\title{
Customizing Immunization Clinic Operations to Minimize Open Vial Waste
}

\author{
Maryam H. Mofrad, Gian-Gabriel P. Garcia, Lisa M. Maillart, \\ Bryan A. Norman, Jayant Rajgopal \\ Department of Industrial Engineering, Swanson School of Engineering \\ University of Pittsburgh, Pittsburgh, PA 15261, USA \\ Telephone: 412-624-9830, Fax: 412-624-9831 \\ mah249@pitt.edu,gig9@pitt.edu, maillart@pitt.edu \\ banorman@pitt.edu,rajgopal@pitt.edu
}

October 19, 2015 


\title{
Customizing Immunization Clinic Operations to Minimize Open Vial Waste
}

\begin{abstract}
Many multi-dose vaccine vials must be used within hours of reconstitution; unused doses are discarded as "open vial waste." Building on Mofrad et al. (2014), we evaluate operating strategies that maximize coverage by controlling open vial waste. We define novel metrics for determining thresholds on vaccination clinic operating hours and session frequency. We study the performance of optimal and heuristic policies in the presence of random vial yield. Cost analyses indicate significant potential savings. Because optimal strategies are context specific, we also develop a decision support tool (available online) to easily replicate the analysis for any problem setting.
\end{abstract}

Keywords: multi-dose vial, vaccine administration policy, open vial waste, vaccine coverage. 


\section{Introduction}

Organized routine immunization programs, especially those in the world's poorest countries, are critical for preventing the incidence of many diseases and for decreasing mortality rates. In many countries (e.g., Niger, India, Bangladesh), a substantial fraction of the clinics or health centers where immunizations occur are in remote locations from which it is difficult to access urban areas. Vaccine wastage is a major issue at such clinics because their stock of vaccines is limited and running out of vaccine before the next replenishment results in missed vaccination opportunities. Reduction in vaccine wastage also ensures the overall financial stability of immunization programs, especially in poor countries with limited budgets for these programs.

Vaccines used in worldwide immunization programs are typically manufactured in two forms: a liquid form that can be directly administered and a freeze-dried powder that must be reconstituted with a diluent before administration. In the powder form, the primary concern prior to reconstitution is the shelf life of the vaccine; it determines the expiration date of the vaccine. After reconstitution, the remaining lifetime of the vaccine is called open vial life, which is considerably shorter than shelf life. For example, a 10-dose vial of MMR vaccine has a 48-month shelf life, but only an 8-hour open vial life (Center for Disease Control and Prevention (2007)). When a multi-dose vial is reconstituted or "opened," but not completely used during its open vial life, the unused doses are discarded. This type of vaccine wastage is called open vial waste (OVW) (Dhamodharan \& Proano (2012)). OVW accounts for a large portion of overall vaccine wastage (Lee et al. (2010)), which averages around 50\% worldwide (World Health Organization (2005)). Vaccines are manufactured in a range of standard vial sizes, e.g., 1, 5, 10, 20 doses per vial. In general, vials with a greater number of doses are less expensive per dose due to their lower production, transportation and storage costs; however, they typically result in higher OVW.

A number of issues must be considered in designing an effective immunization program 
with low wastage. The majority of these issues are addressed at higher levels of decision making within governmental immunization organizations, e.g., determining the best vial size; designing the distribution chain; setting storage capacities, replenishment frequencies, and order quantities at various levels of the vaccine supply chain; and deciding on transportation modes and their capacities. Vial size and inventory considerations in particular have been examined in several previous studies. Parmar et al. (2010), Lee et al. (2010), Lee et al. (2011) and Yang et al. (2014) perform economical analysis to determine the appropriate vial size. More specifically, Parmar et al. (2010) estimate the potential wastage cost associated with different vial sizes in various countries. Lee et al. (2010) show that the most economical vial size is a function of mean daily demand (i.e., vials with a greater number of doses produce less OVW as the mean daily demand increases). Lee et al. (2011) determine a threshold on the mean daily demand for the adoption of a specific vial size; they also argue that using single-dose vials (which are physically larger on a per-dose basis) can severely constrain transportation capacity in the vaccine distribution supply chain and result in increased risk of vial breakage. Lastly, Yang et al. (2014) investigate the economical impact of vial size using empirical data. Related to this work, Assi et al. (2011) study the impact of different vial sizes on the vaccine supply chain. Lastly, Dhamodharan \& Proano (2012) integrate an optimization model and simulation to simultaneously determine vial size and ordering policy under the assumption of $100 \%$ coverage. Regardless of how vial size and replenishment frequency are determined, questions surrounding the downstream issue of how to best administer doses from multi-dose vials remain open.

In this paper, we focus on the lowest (i.e., clinic) level of the vaccine distribution chain, especially those in remote locations. Specifically, we focus on the development of vaccine 
administration policies when using multi-dose vials at these clinics. Most clinics currently operate under a policy that never turns away a patient as long as the requested vaccine is available; we refer to this policy, which typically results in high OVW, as the greedy policy. For example, if a patient arrives just as the clinic is closing and a new 20-dose vial is reconstituted, it is highly likely that 19 of these doses will be discarded. Thus, this type of myopic approach can result in significant OVW, which in turn can lead to missed opportunities for vaccinations. Improving the effectiveness of immunization programs requires tailored clinic operations and smarter vaccine administration policies that specifically address missed opportunities caused by a shortage of vaccines due to high OVW.

As previously stated, the existing literature on multi-dose vials is rather limited, and primarily focuses on the economic implications of single- vs. multi-dose vials. In contrast, Mofrad et al. (2014) address another means for controlling OVW, namely that of vaccine administration from multi-dose vials, using a rigorous mathematical approach. They formulate a Markov decision process (MDP) model that maximizes the number of vaccinations between two consecutive vaccine stock replenishments (i.e., over one "replenishment cycle") by determining when to discontinue vaccinations as a function of time of day, the current vial inventory and the remaining number of vaccination sessions until the next inventory replenishment. We refer to such a strategy as a "vaccine administration policy." (Note that by "sessions" we mean the number of dedicated periods per cycle during which the clinic operates; a session typically corresponds to a working day, e.g., an eight hour session that runs from 8am-4pm.) Although they consider the same decision making problem considered herein, the focus in Mofrad et al. (2014), is on model formulation, policy structure and limited sensitivity analysis under the single objective of maximizing the mean number of vaccinations administered.

The contributions of this paper are five-fold. First, we pair combined analysis of the MDP model with simulation to perform descriptive analysis of the dis- 
tribution of session duration induced by an optimal administration policy. We examine this novel metric of policy performance because a coverage-maximizing/ waste-minimizing administration policy that induces large variability in a clinic's hours of operation may inconvenience patients and lead to undesirable long-term consequences. Second, we explore means by which a clinic can directly control patient convenience by imposing a minimum number of guaranteed hours per session or increasing the session frequency. We explore how these two means of control interact with each other as well as when the latter can counterintuitively affect coverage and wastage. Third, we propose a novel, easy to implement static heuristic policy that induces zero variability in session duration and compare its performance to that of two other heuristic policies. Fourth, we introduce the concepts of random vial-yield and vial failures to this problem setting and assess their impact on the performance of the optimal and heuristic policies. Lastly, we use data available for three countries to perform novel costs analyses for a single vaccine over all GAVI countries, which suggest potential savings on the order of $\$ 4.6$ million.

The remainder of the paper is organized as follows. In Section 2, we provide an overview of the MDP model developed in Mofrad et al. (2014) and a new simulation model created to evaluate additional performance metrics of interest. In Section 3, we conduct extensive computational analyses to generate insights on the relationships between day-to-day clinic operations and the vaccine administration policy. In Section 4, we present our heuristic policy analysis. We then, in Section 5, study the performance of the optimal policy and the heuristic policies in the presence of vial failures and random vial yield. In Section 6, we summarize the results in the form of some general operational recommendations based on the analysis in Sections 3 and 5 and estimate the procurement cost savings realized by switching to the optimal policy. Lastly, 
in Section 7, we discuss limitations of the work and possible future extensions.

\section{Overview of Models}

In Section 2.1, we describe the MDP model developed in Mofrad et al. (2014), which is used to generate optimal vaccine administration policies. In Section 2.2, we introduce a new simulation model that is used to simulate the performance of a clinic under a given vaccine administration policy generated by the MDP model.

\subsection{Markov Decision Process Model}

To clarify the modeling approach used in Mofrad et al. (2014), we present Figure 1. Mofrad et al. (2014) develop a finite horizon MDP model for vaccination sessions between two stock replenishments where $Q$ vials are available at the beginning of each replenishment cycle and each vial consists of $z$ doses. As seen in Figure 1, the replenishment cycle is divided into $T$ sessions and each session is divided into $\eta$ timeslots of equal length. At each point in time, the state of the system is given by $t$, the number of sessions remaining until the next replenishment, $q$, the number of remaining vials and $h$, the current timeslot.

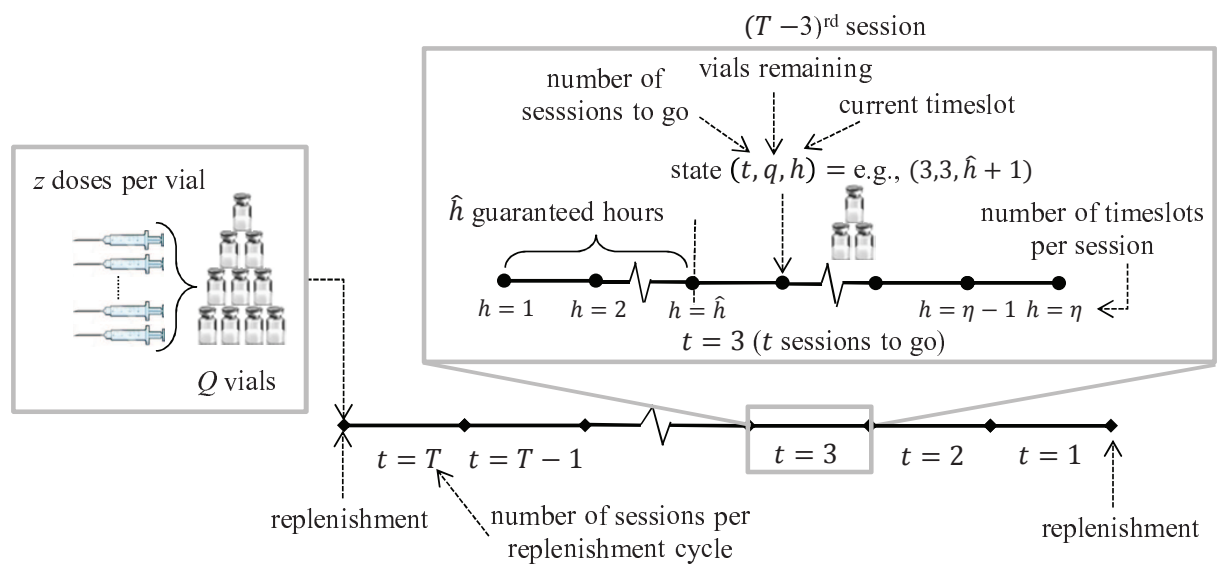

Figure 1: Replenishment cycle timeline.

The MDP model determines when to discontinue opening new vials during a session 
as a function of the time of day, the current vial inventory and the remaining number of sessions until the next inventory replenishment. Mofrad et al. (2014) show that the MDP model results in an optimal vaccine administration policy that is of a threshold type. For convenience, we refer to this threshold as the clinic "closing time," although it is worth noting that the clinic will actually continue to vaccinate whenever there is a vial open and will remain open as long as other activities are being performed at the clinic. The model objective is to maximize the number of vaccinations administered over the replenishment cycle, given an initial inventory of vaccine vials. This objective results in low OVW while achieving the highest level of coverage, i.e., percentage of demand satisfied.

In the formulation of the finite horizon MDP model, the following assumptions are made (see Mofrad et al. (2014) for more details):

- there is a single type of vaccine,

- the open vial life is greater than or equal to the maximum number of working hours per session (Center for Disease Control and Prevention (2007) and Merck \& Co. (2014)),

- the mean daily demand is constant throughout the replenishment cycle and is not affected by the clinic discontinuing service before the end of the scheduled operating hours,

- the maximum number of patient arrivals per timeslot is one.

The model produces a vaccine administration policy that yields a time of day for each session, beyond which a new vial will not be opened. This time, $h^{*}(t, q)$, is a function of the current vial inventory level $(q)$ and the remaining number of sessions until the next replenishment $(t)$. As shown in Figure 2, the policy depends on the vial size in doses $(z)$, the initial number of vials for the replenishment cycle $(Q)$, the number of sessions between two successive replenishments $(T)$, the mean daily demand from the catchment population covered by the clinic $(\mu)$ and the minimum number of hours per session, i.e., guaranteed hours $(\hat{h})$. 


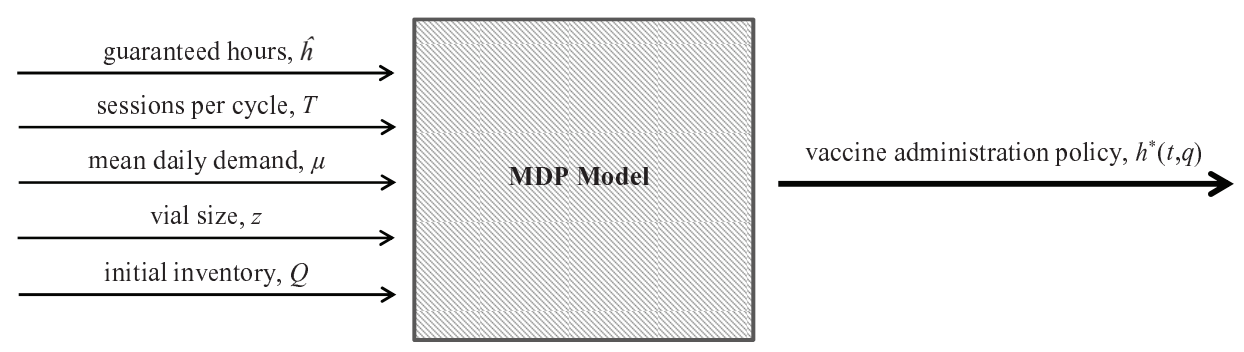

Figure 2: MDP model inputs and output.

\subsection{Simulation Model}

In this section, we present a simulation model used to assess the performance of the vaccine administration policies produced by the MDP model. This model simulates a clinic that administers one type of vaccine over one replenishment cycle. Similar to the MDP model, sessions are divided into $\eta=480$ timeslots, and arrivals occur at the beginning of each timeslot according to a Bernoulli distribution with probability $\frac{\mu}{\eta}$.

A flowchart of the simulation model is shown in Figure 3. In each replication, the clinic starts at the beginning of the replenishment cycle with $Q$ vials on hand. Arrivals occur randomly in each timeslot and $h$ denotes the current timeslot. Whenever an open vial is available, clinicians vaccinate arriving patients. Whenever an arrival occurs and no vial is open, the decision to open a new vial is made according to the vaccine administration policy, $h^{*}(t, q)$. Hence, clinicians discontinue service at the first timeslot greater than $h^{*}(t, q)$ when no open vial is available. As mentioned in Section 2.1, unserved patients are lost and leave the system with no effect on future demand. Note that the simulation of each session extends beyond the time that the clinic discontinues service so that the number of unserved patients can be tallied.

\section{Impact of Clinic Operations on Policy Performance}

As explained in Section 2.1, the MDP model maximizes the expected number of vaccinations under a given set of parameter values. In this section, we analyze how the parameters 


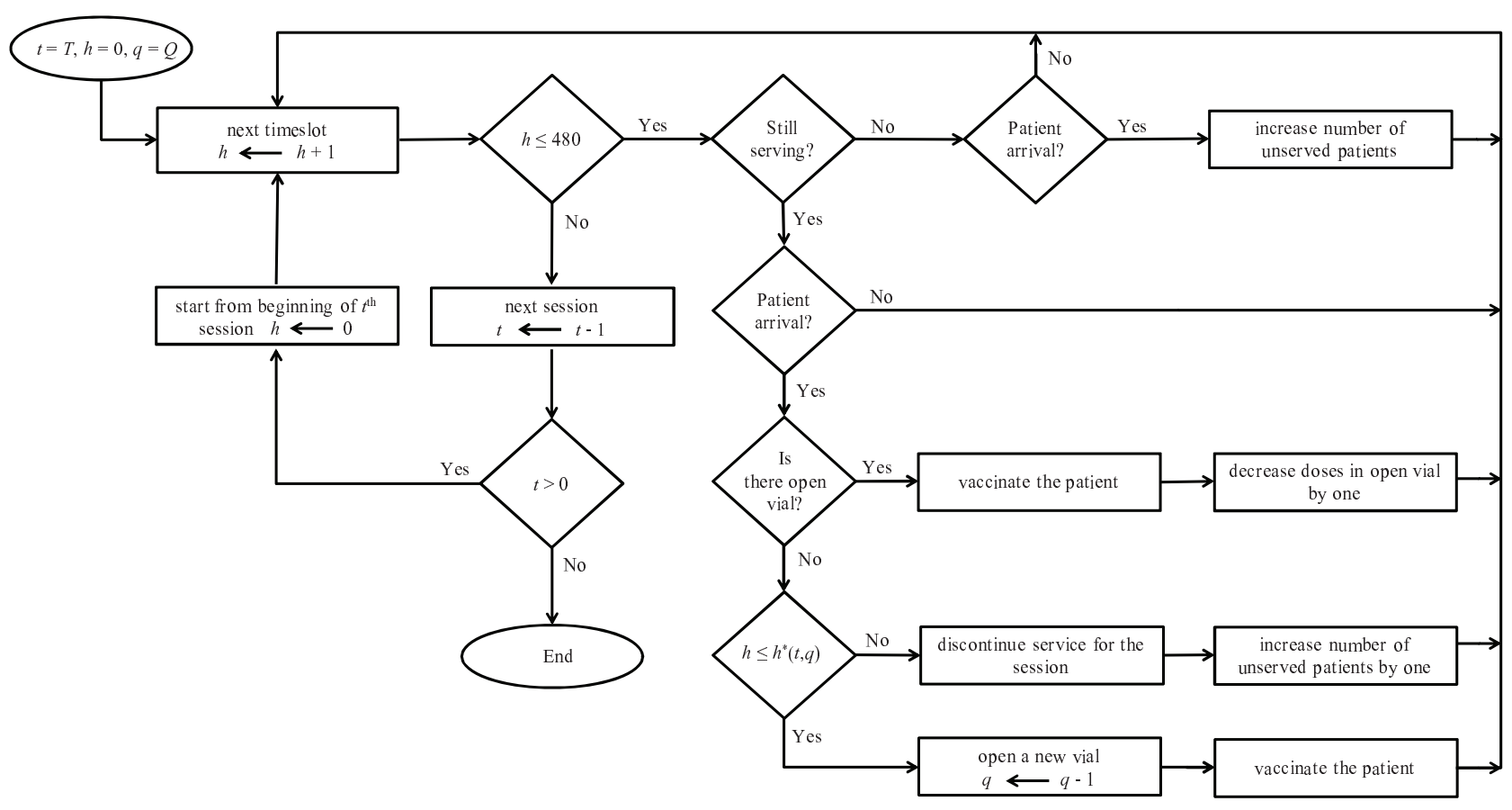

Figure 3: Simulation model flowchart.

under the clinic's control (the number of vaccination sessions per replenishment cycle and guaranteed hours per session) interact with parameters that the clinic does not typically control (e.g., vial size, replenishment interval and initial inventory) to determine policy performance. We assess these relationships and their inherent trade-offs as a function of coverage rate, OVW and additional new metrics (e.g., closing time distribution, prediction intervals on the number of open hours, and percentage gain/loss compared to benchmark vaccine administration policies).

More specifically, in Section 3.1, we analyze session duration under the simplest form of the optimal policy (i.e., one with no set number of guaranteed hours). That is, we evaluate, via simulation, how session duration changes as a function of the number of sessions per cycle and observe that in some cases, considerable variation exists from session to session within a replenishment cycle. Such variability could create operational challenges. Hence, in 
Section 3.2, with the aim of preventing discontinuation of vaccine administration "too early" during a session, we consider imposing some minimum number of working hours per session and propose a metric for determining the most desirable duration of these guaranteed hours. In Section 3.3, we evaluate the effect of the number of sessions per replenishment cycle on the performance of the optimal policy while operating the clinic for a specified number of guaranteed hours (i.e., using the results of Section 3.2). In doing so, we focus on determining the appropriate number of sessions per replenishment cycle in order to achieve a coverage target under an optimal policy with guaranteed hours. The goal is to offer as many sessions as possible to facilitate patient access to the clinic, without compromising coverage.

The main criterion used to evaluate the performance of a vaccine administration policy is the expected number of vaccinations per cycle, i.e.,

$$
\text { coverage rate }=\frac{\text { expected number of vaccinations }}{\text { expected number of arrivals }}
$$

That is, by coverage rate, we mean the expected percentage of patients who arrive and are vaccinated.

Throughout this section, we consider values of $T \in\{4,8,12,16,20\}$, which are equivalent to 1-5 sessions per week for a one-month replenishment cycle; values of $z \in\{1,5,10,20\}$ which represent the most common vial sizes; and set the initial inventory to:

$$
Q=1.25 \frac{\mu T}{z}
$$

which corresponds to a $25 \%$ buffer stock, i.e., the clinic starts each replenishment cycle with $25 \%$ more doses than the expected cycle demand, $\mu T$. These ranges represent values commonly seen in practice (Lee et al. (2010), Drain et al. (2003)). Furthermore, consistent with Mofrad et al. (2014), in the remainder of the paper we assume that the number of sessions per cycle does not significantly affect the distribution of the demand between two 
stock replenishments. That is, in comparing different values of $T$, we assume that $\mu$ scales accordingly so that $\mu T$ remains constant. We consider two values for the expected number of arrivals per cycle, namely $\mu T=96$ and $\mu T=288$. These are typical values observed in practice for medium-size and large clinics, respectively, for a two-dose vaccine schedule (see Section 6.2). Note that to maintain consistency in buffer stock across all of the problem instances considered, both of these values always result in an integer value for $Q$, the initial vial inventory, as defined by equation (2).

\subsection{Session Duration}

We refer to the optimal vaccine administration policy with no requirements on the minimum number of working hours per session (i.e., $\hat{h}=0$, where $\hat{h}$ is the earliest timeslot in which the clinic may elect to discontinue service) as the "unrestricted policy." In this section, we first analyze the frequency with which it is optimal to discontinue service early under the unrestricted policy as a function of the number of sessions per cycle for various vial sizes. We then investigate the effect of the number of sessions per cycle on the full distribution of the closing time and the variability of the number of operating hours per session under the unrestricted policy.

Based on 10,000 replications of the simulation model, the black regions in Figure 4 indicate the percentage of sessions that terminate before the end of the scheduled operating hours under the unrestricted policy. As can be seen, a larger vial size generally results in a higher frequency of early termination. This behavior can be explained by the fact that, on average, a smaller vial size results in less OVW, and therefore higher inventory levels

throughout the replenishment cycle, which permits the clinic to remain open longer. (The exception to this observation is the $(z=20, T=20)$ case compared to the $(z=10, T=20)$ case. Note, however, that the $z=20, T=20$ case is somewhat extreme in that the initial inventory is so low compared to the number of sessions that the clinic is unable to open a 
vial in each of the 20 planned sessions.)

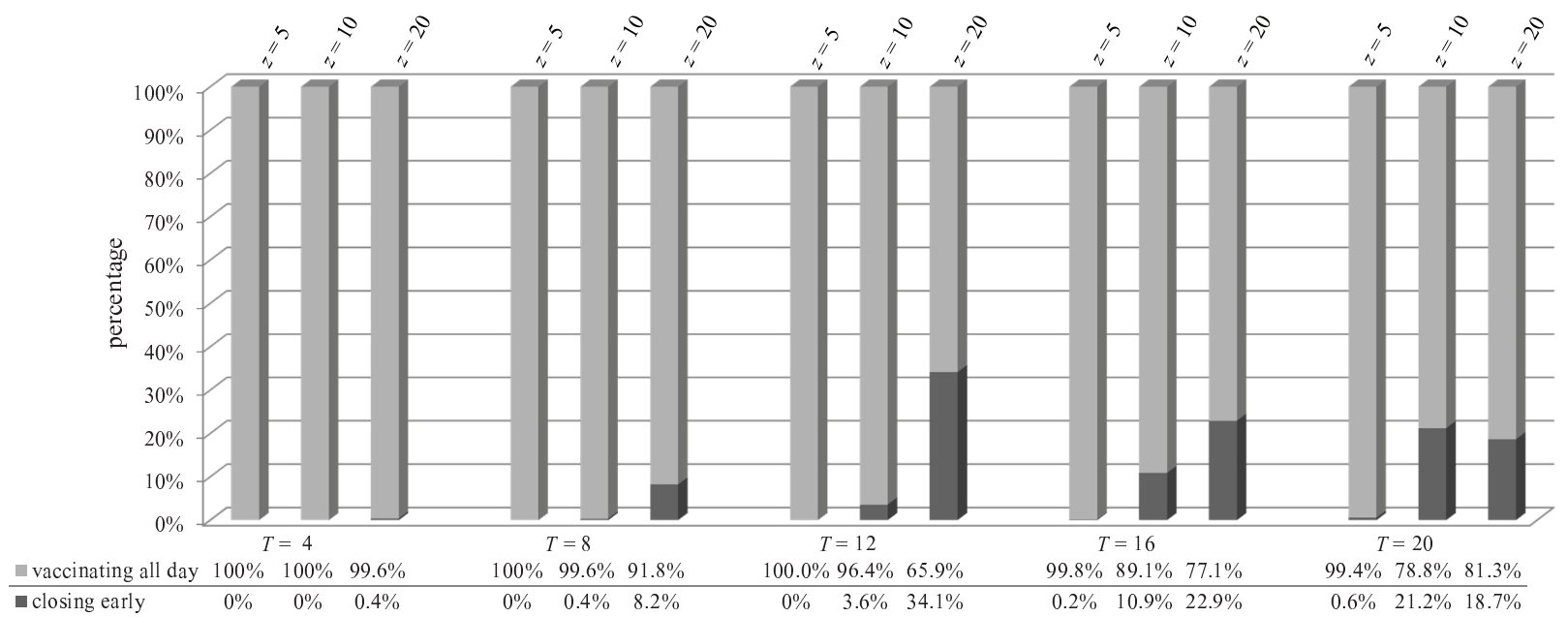

Figure 4: Percentage of sessions that terminate before the end of the scheduled operating $\operatorname{hours}(z Q=360, \mu T=288$ and $\hat{h}=0)$.

Interestingly, however, Figure 4 suggests that the frequency of early closure is not necessarily monotone in the number of sessions per cycle. For $z=20$, the percentage of sessions that terminate early is larger for $T=12$ than for $T \in\{8,16,20\}$. This behavior is somewhat counterintuitive because increasing the number of sessions generally increases opportunities for OVW and hence entices the clinic to act more conservatively by closing early. To explain this behavior, consider the ratio $\frac{Q}{T}$, i.e., the "number of vials per session" if the vials are divided equally across the sessions. Intuitively, the vaccine administration policy is expected to result in fewer early closures when this ratio is large (plenty of inventory) and/or close to an integer value (an integral number of vials are needed per session, in expectation). For example, for $z=20$ in Figure 4, the number of vials per session is 1.5 for $T=12$ sessions, whereas the number of vials per session is 1.1 for $T=16$ sessions. Although offering 4 more sessions, when $T=16$ a smaller percentage of these sessions terminate early because each session is likely to need one (and only one) vial to satisfy demand. When $T=12$, on the other hand, there is a good chance that each session will need a second vial, but that much 
of this vial will go unused if opened, resulting in the optimal policy dictating more frequent early closures.

Based on the same simulation data, Figure 5 explores the entire distribution of the closing time, as opposed to simply whether or not the closing time occurs before the scheduled session end time, under the unrestricted policy. Because a large percentage of the simulated sessions operate for the fully planned duration (see Firgure 4), Figures 5(a) and 5(b) plot the closing time distribution conditional on terminating the vaccination session before the end of the scheduled operating hours.

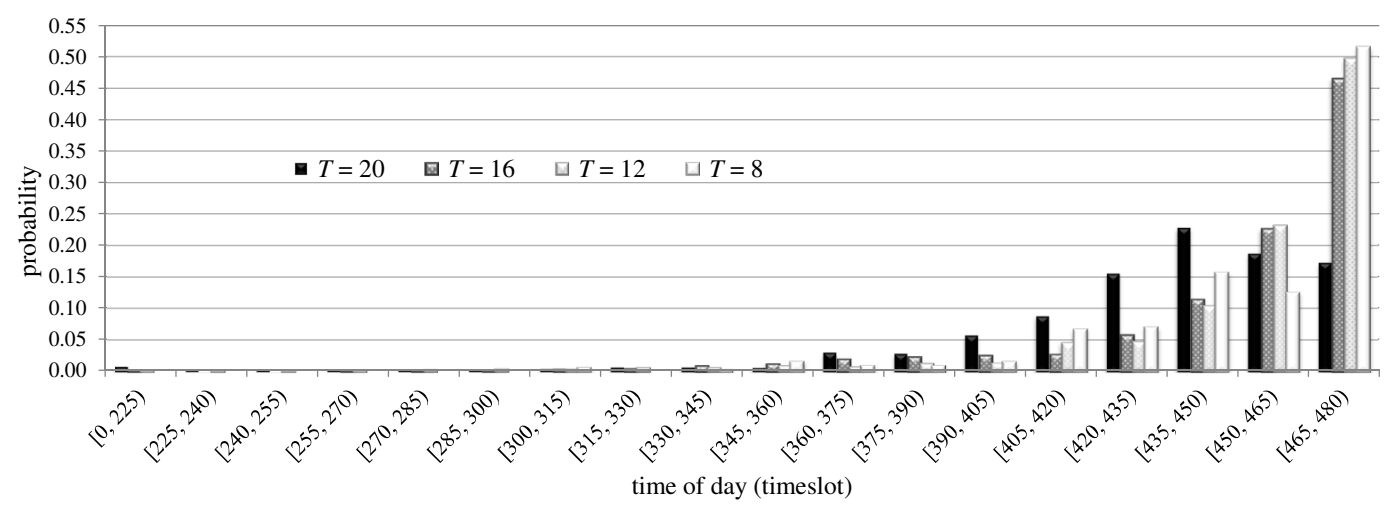

(a) $z=10, Q=36, \mu T=288, \hat{h}=0$.

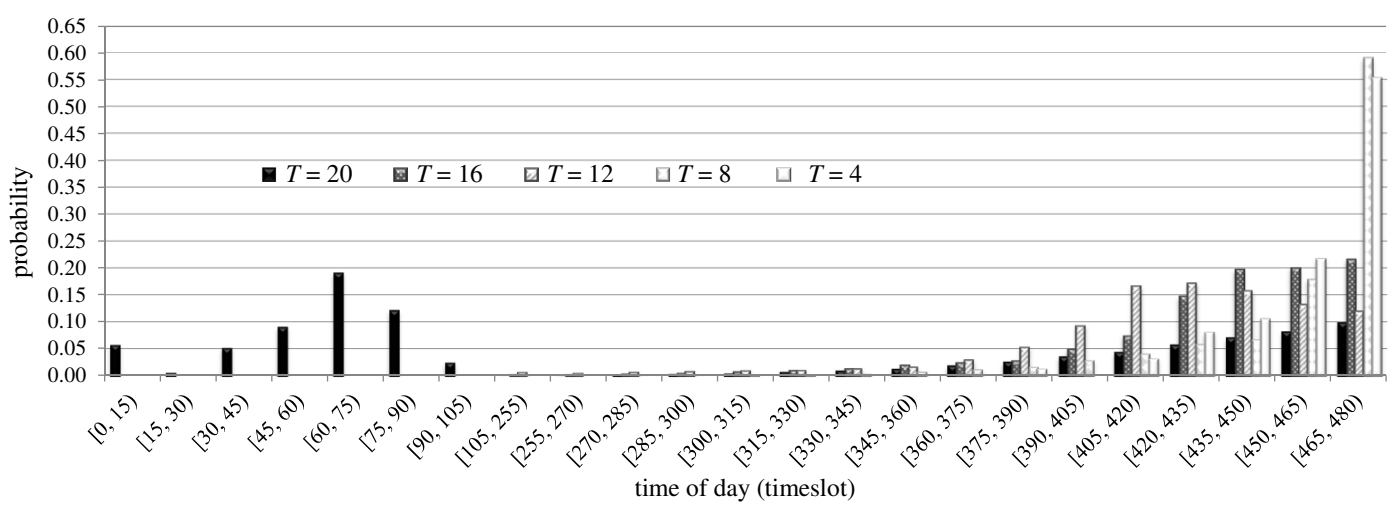

(b) $z=20, Q=18, \mu T=288, \hat{h}=0$.

Figure 5: Closing time distribution conditional on discontinuing service before the end of the scheduled operating hours.

Based on Figures 5(a) and 5(b), the closing time distribution increases monotonically in 
the time of day for fewer sessions per cycle, whereas this behavior is not monotonic for a greater number of sessions per cycle. For example, in Figure 5(a), for $T=20$ the frequency of closing early becomes significant after timeslot 360 (e.g., 2pm for an 8am-4pm session). After timeslot 360, the distribution increases up to timeslot 450 (e.g., 3:30 pm for an 8am4pm session), at which point it attains its highest value and then decreases. That is, early closing times tend to occur earlier in the day (though generally less often) when the number of sessions per cycle is larger. This non-monotonic behavior occurs because at the beginning of the cycle under larger $T$, there is a higher chance that preserving vials for future use results in a larger number of vaccinations in the subsequent sessions. Therefore, the optimal policy is more conservative regarding opening the last vial at the beginning of replenishment cycle and as a result, an earlier closing time occurs more frequently. Indeed, for the extreme case $(T=20)$ in which the initial number of vials is less than the number of sessions in Figure 5(b), very early closures are not uncommon. Very early closures occur when the first arrival occurs relatively late in the day, in which case it is optimal to retain all vials for later sessions in which the first arrival is likely to occur earlier in the day resulting in a higher chance of vaccinating more patients.

Next, we consider the variability of the hours of operation under the unrestricted policy by constructing prediction intervals. Clearly, larger variability is undesirable from a patient perspective. The number of hours open is bounded above by the maximum number of hours per session. Therefore, to characterize the variability of the operating hours we construct a 99\% one-sided prediction interval based on 10,000 replications of the simulation. Figure 6 reports the resulting prediction intervals for several problem scenarios. Generally speaking, the mean (respectively, the variance) of the number of operating hours decreases (respectively, increases) in the number of sessions per cycle. Therefore, for a larger number of sessions, the closing time is less predictable, which results in potentially more inconvenience for patients. In Figure 6(a), the variability is strictly increasing in the number of sessions 
per cycle, whereas in Figure 6(b), the variability is not monotonic. In addition to having a higher percentage of sessions that terminate before the end of the scheduled operating hours (see Figure 4), the $T=12$ scenario in Figure 6(b) has more variability in the conditional number of hours open than $T=8$ and $T=16$. That is, for the case with $\frac{Q}{T}=1.5$, not only does the optimal policy result in more frequent early closures, but these early closure times are more erratic. (The dramatic increase in the width of the prediction interval for $T=20$ is again due to the fact that for this scenario, the initial number of vials is less than the number of sessions, resulting in at least two sessions with zero operating hours.)

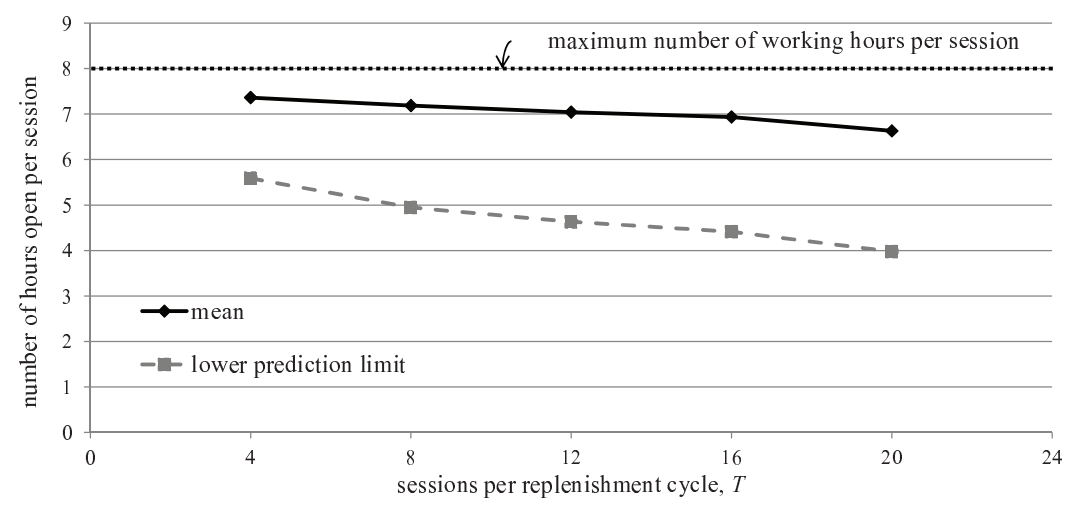

(a) $z=5, Q=24, \mu T=96, \hat{h}=0$.

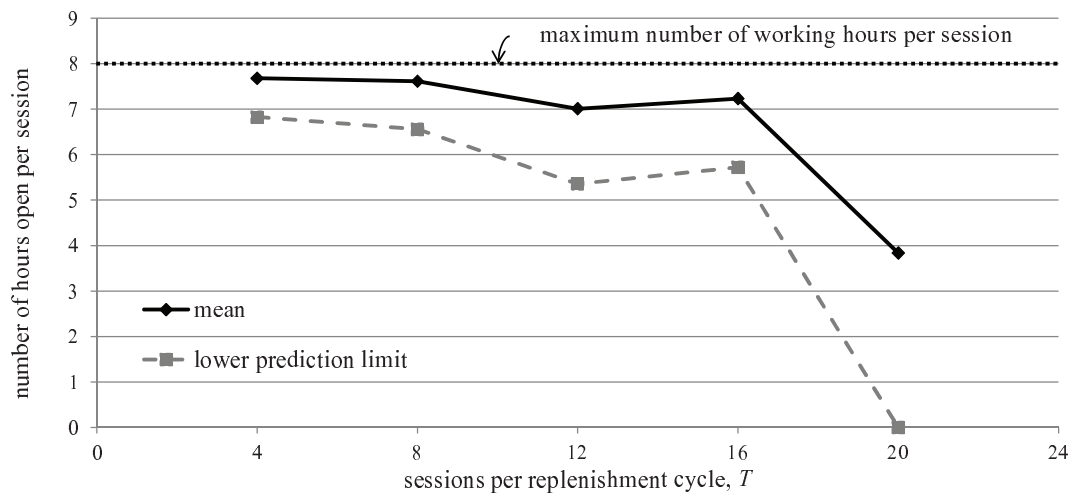

(b) $z=20, Q=18, \mu T=288, \hat{h}=0$.

Figure 6: 99\% prediction interval on the number of hours open per 8-hour session, conditional on discontinuing service before the end of scheduled operating hours.

Unpredictable closing times can be inconvenient, especially in a developing 
country in which travel logistics to reach a vaccination site can be difficult for a large portion of the population. This unpredictability motivates the need to define a fixed minimum number of working hours per session during which clinicians vaccinate unconditionally. In the following section, we investigate the impact of imposing such guaranteed hours on the vaccine administration policy.

\subsection{Guaranteed Hours per Session}

Mofrad et al. (2014) introduce the concept of a minimum number of working hours or "guaranteed hours" per session, i.e., an earliest closing time, in order to decrease the inconvenience associated with unpredictable operating hours. Their analysis of this parameter, however, is limited in scope. Hence, in this section, we analyze the performance of the "restricted policy" (i.e., the optimal vaccine adminstration policy under a required minimum number of working hours per session) as a function of the number of guaranteed hours per session. We also propose a metric that can be used to determine an appropriate number of guaranteed hours per session based on an acceptable percentage drop in the performance of the restricted policy versus the unrestricted policy.

Let $\nu(T, Q, \hat{h})$ be the expected number of vaccinations administered between two stock replenishments over $T$ sessions, with $Q$ initial vials and $\hat{h}$ guaranteed hours per session (expressed in timeslots, e.g., $\eta=480$ corresponds to the maximum number of working hours per session, say 8 , in which case $\hat{h}=240$ corresponds to a minimum of four hours per session). Furthermore, let $\gamma(T, Q, \hat{h})$ be the percentage gain in the expected number of vaccinations under the restricted vaccine administration policy versus the greedy policy (as described in Section 1) over $T$ sessions, with $Q$ initial vials and $\hat{h}$ guaranteed hours per session, i.e.,

$$
\gamma(T, Q, \hat{h})=\frac{\nu(T, Q, \hat{h})-\nu(T, Q, \eta)}{\nu(T, Q, \eta)} \times 100
$$

Figure 7 parts (a) and (b) report the percentage gain as a function of guaranteed hours 
per session based on the expected number of vaccinations as determined by the MDP model. As seen in these figures, a sharp drop occurs in the performance of the restricted policy for large $\hat{h}$. This drop in performance is caused by the fact that imposing a large number of guaranteed hours may require clinicians to open new vials close to the end of the day, which are then not used completely. Prior to this point, however, the curves are relatively flat, indicating that offering patients the convenience of a reasonable number of guaranteed hours does not significantly degrade policy performance. Furthermore, as anticipated based on Figure 4, imposing guaranteed hours does not significantly affect the performance of the vaccine administration policy for smaller vial sizes because the optimal policy does not typically stop vaccinating until the end of the session as planned. When larger vial sizes are used, defining the number of guaranteed hours is more critical, as doing so can negatively impact the performance of the vaccine administration policy.

A reasonable value of the minimum number of working hours per session would guarantee acceptable performance of the vaccine administration policy without eroding patient convenience. To explore this trade off, let

$$
\hat{h}^{*}(T, Q, \alpha \%)=\max \left\{30\left\lfloor\frac{\hat{h}}{30}\right\rfloor: \frac{\gamma(T, Q, 0)-\gamma(T, Q, \hat{h})}{\gamma(T, Q, 0)} \leq \alpha \%, 0 \leq \hat{h} \leq \eta\right\}
$$

That is, let $\hat{h}^{*}(T, Q, \alpha \%)$ be the greatest number of guaranteed hours per session (rounded down to the nearest 30 minute increment to avoid unnatural closing times) that results in at most an $\alpha \%$ drop in performance compared to the unrestricted vaccine administration policy. The dashed circles in Figure 7 indicate $\hat{h}^{*}(T, Q, 1 \%)$ for each scenario considered. For example, in Figure $7(\mathrm{a}), \hat{h}^{*}(16,36,1 \%)=360$, i.e., an earliest closing time of $2 \mathrm{pm}$ for a clinic that operates from $8 \mathrm{am}-4 \mathrm{pm}$. Throughout the remainder of the paper, we suppress dependence on T, $Q$ and $\alpha$ for notational convenience and let $\hat{h}^{*} \equiv \hat{h}^{*}(T, Q, 1 \%)$.

In Figure 8, we plot $\hat{h}^{*}$ as a function of the number of sessions per replenish- 


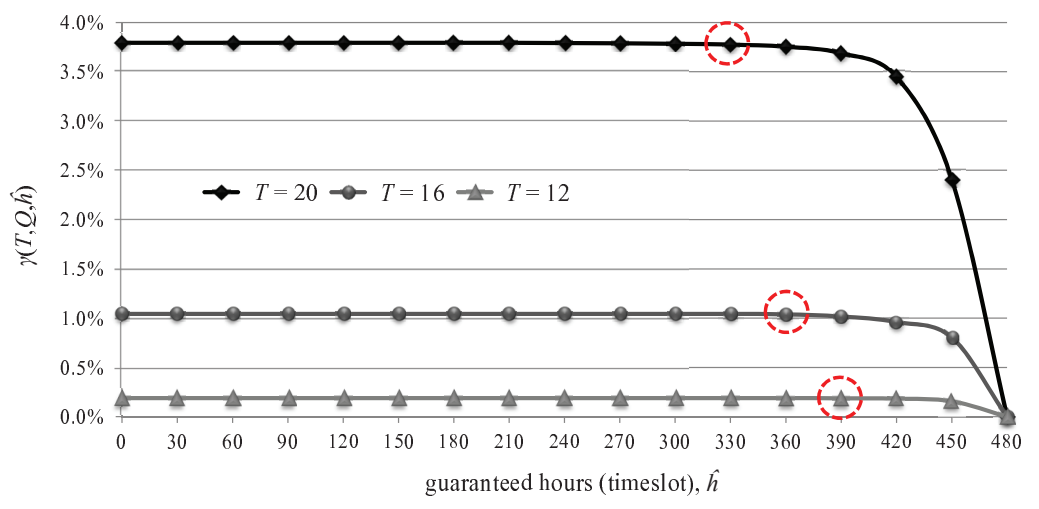

(a) $z=10, Q=36, \mu T=288$.

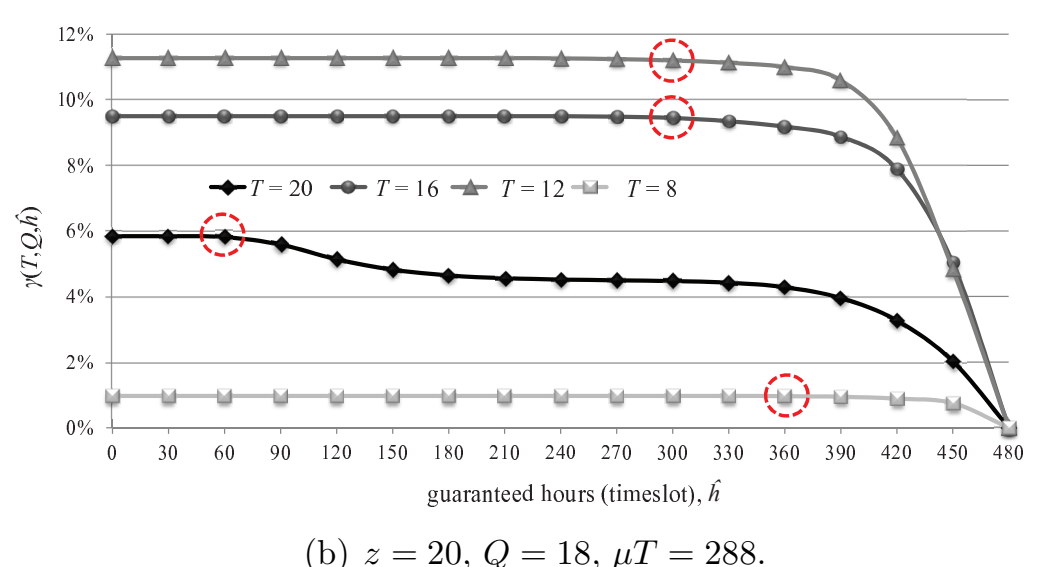

Figure 7: Percentage gain in expected number of vaccinations versus the greedy policy as a function of guaranteed hours.

ment cycle. The value of $\hat{h}^{*}$ generally decreases in T because the arrival rate per timeslot decreases in $T$ and to prevent high OVW, it is optimal to discontinue vaccination earlier in the day. However, there are ranges of $T$ over which $\hat{h}^{*}$ is nonmonotone (e.g., $T=5$-10 for $z=20$ ) because the ratio $\frac{Q}{T}$ becomes far an integer value and the optimal policy is such that it is likely to close early in the day. Therefore, smaller values of $\hat{h}$ satisfy the inequality in equation (4). Finally, as vial size increases, $\hat{h}^{*}$ decreases because a larger vial size results in higher OVW and, consequently, requires a more flexible guaranteed hours policy. 


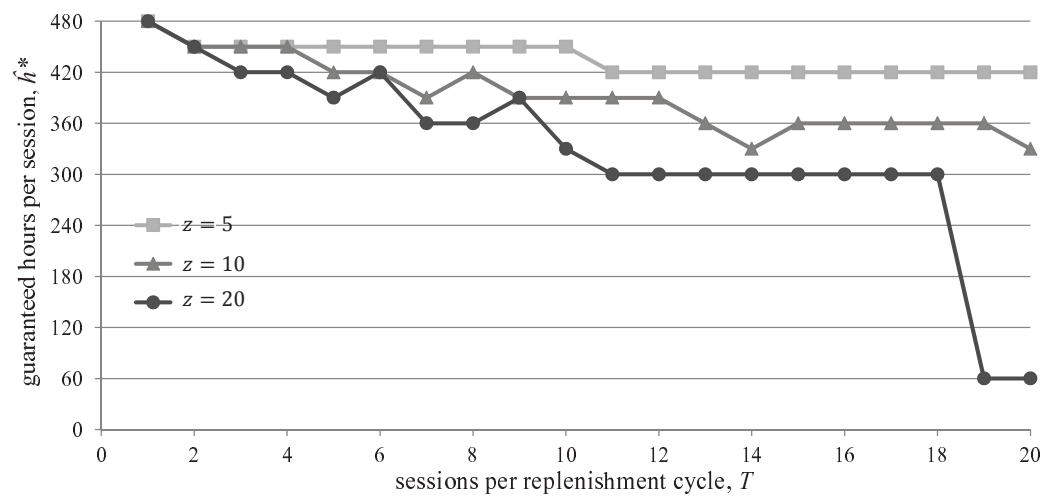

Figure 8: Value of $\hat{h}^{*}$ as a function of number of sessions per replenishment cycle $(z Q=360$ and $\mu T=288)$.

\subsection{Vaccination Session Frequency}

Next, we investigate the degree to which the number of sessions per cycle can be increased, providing more days of clinic access to patients, without significantly impacting the coverage rate. Note that similar analysis can be performed to find the number of sessions per cycle that minimizes OVW; because our results indicate that these two values of $T$ are typically close if not the same, we present the results for coverage only.

To evaluate the effect of the number of sessions per cycle on the coverage rate, we define the criterion $\beta(T, Q, \hat{h})$ as the percentage loss in the expected number of vaccinations administered over $T$ sessions with $Q$ vials initially on hand and $\hat{h}$ guaranteed hours (in timeslots), as compared to that under the administration policy with the same initial inventory, but only one session per replenishment cycle (i.e., $T=1$ ) and guaranteed hours of $\eta$, i.e.,

$$
\beta(T, Q, \hat{h})=\frac{\nu(1, Q, \eta)-\nu(T, Q, \hat{h})}{\nu(1, Q, \eta)} \times 100
$$

That is, $\beta(T, Q, \hat{h})$ compares the performance of the optimal restricted vaccine administration policy to the idealized policy where the clinic is open for exactly one full session between 
two stock replenishments. For a fixed demand per cycle, the latter policy has the highest coverage rate of all values of $T$ and the lowest OVW. Similar to equation (4) for determining the number of guaranteed hours, this criterion can be used to determine the greatest number of sessions that results in an at most $\alpha \%$ loss in coverage as compared to the single-session case, denoted $\hat{T}(Q, \hat{h}, \alpha \%)$, using

$$
\hat{T}(Q, \hat{h}, \alpha \%)=\max \{T: \beta(T, Q, \hat{h}) \leq \alpha \%, 0 \leq T \leq \bar{T}\}
$$

where $\bar{T}$ is the number of calendar days between two replenishments. For example, in Figure $9(\mathrm{~b})$, for $z=20, \hat{T}(18,390,1 \%)=9$ sessions per cycle.

Figure 9 plots the percentage loss as defined in equation (5) as a function of the number of sessions based on the expected number of vaccinations as determined by the MDP model. Note that the percentage loss is not necessarily monotone in the number of sessions. This complicated behavior is related to the ratio of the initial inventory over the number of sessions, as defined in Section 3.1. In Figure 9(b), for $z=20(Q=18)$, the percentage loss increases considerably for $9<T \leq 12$ and then decreases up to $T=18$, after which there is a sharp rise. In the range $9 \leq T \leq 12$ the difference between the ratio $\frac{Q}{T}$ and its nearest integer increases; in the range $12 \leq T \leq 18$, this difference decreases. Lastly, for $z=20$ and more than 18 sessions, the performance of the vaccine administration policy drops quickly, because the number of initial vials is less than the number of sessions.

The better performing ratios of $\frac{Q}{T}$ correspond to problem instances that strike a balance between minimizing expected OVW and having additional vials to satisfy peak demands. For example, the coverage for $T=17$ is higher than for $T=16$ because there is less expected lost coverage for each period in which exactly one vial is opened. The benefit of $T=16$ having two additional vials to meet peak demand days, versus the one additional vial of $T=17$, is not enough to overcome the expected lost coverage on the other 14 days when 


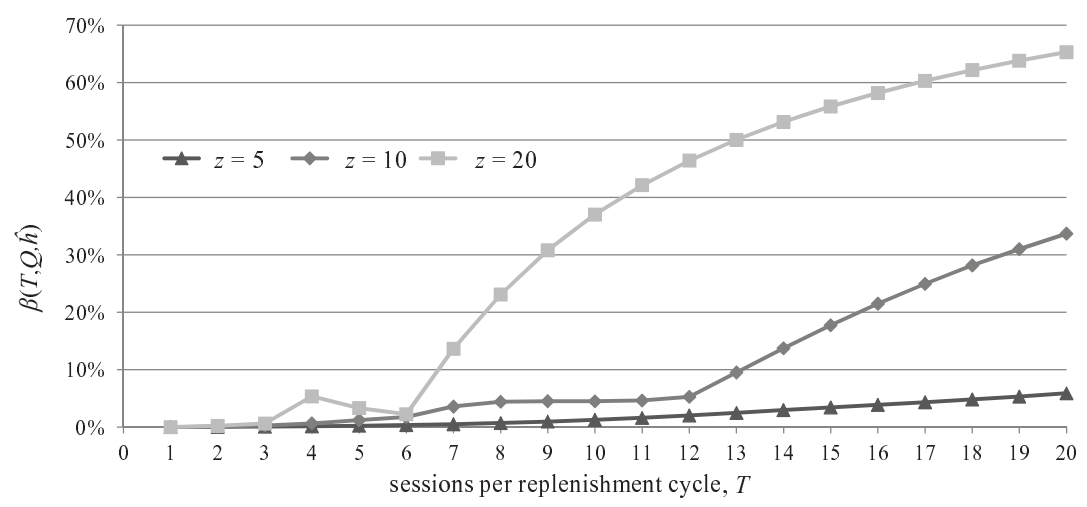

(a) $\mu T=96, Q z=120, \hat{h}=\hat{h}^{*}$.

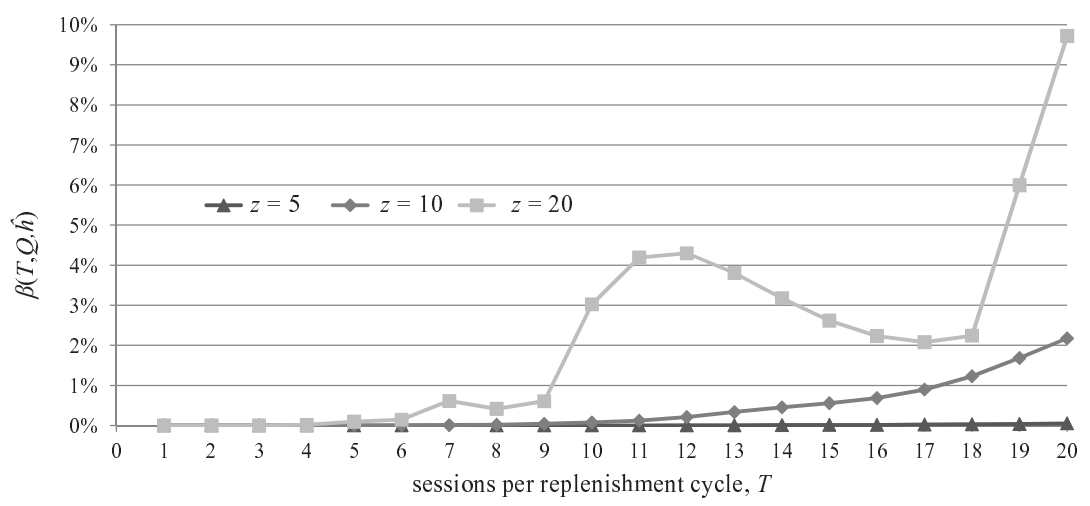

(b) $\mu T=288, Q z=360, \hat{h}=\hat{h}^{*}$.

Figure 9: Percentage loss in the expected number of vaccinations administered $\beta(T, Q, \hat{h})$ as a function of number of sessions per cycle.

only one vial can be used.

Next, we study the impact of the initial inventory or equivalently the buffer stock, on $\hat{T}\left(Q, \hat{h}^{*}, 1 \%\right)$. To do so, for a fixed set of initial inventories, we first compute $\hat{h}^{*}$ for potential values of $T$ and then find the corresponding value of $\hat{T}\left(Q, \hat{h}^{*}, 1 \%\right)$. In Figure 10, we plot $\hat{T}\left(Q, \hat{h}^{*}, 1 \%\right)$ and the total number of guaranteed hours (in timeslots) as a function of the initial inventory. As $Q$ increases, $\hat{T}\left(Q, \hat{h}^{*}, 1 \%\right)$ increases because more buffer stock is available to mitigate the increased OVW associated with a larger number of vaccination sessions. On the other hand, although increasing the number of sessions results in fewer guaran- 
teed hours per session, the total number of guaranteed hours over the replenishment cycle generally increases in $Q$.

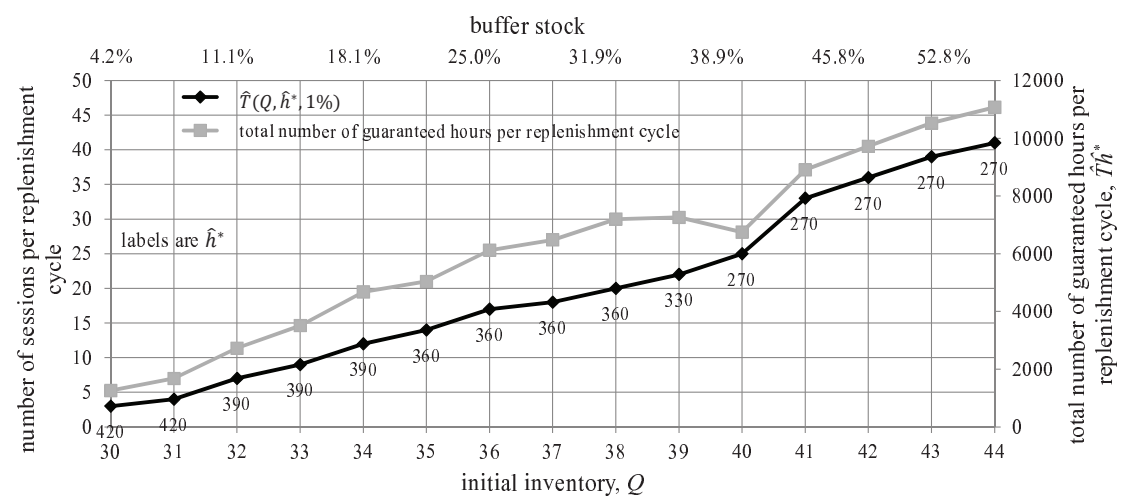

Figure 10: The value of $\hat{T}\left(Q, \hat{h}^{*}, 1 \%\right)$ and its corresponding number of guaranteed hours per replenishment cycle as a function of initial inventory $(z=10, \mu T=288$, $\bar{T}=\infty$ and $\left.\hat{h}=\hat{h}^{*}\right)$.

Additionally, in Figure 11 we plot the "most convenient" pair of $(\hat{h}, \hat{T}(Q, \hat{h}, \alpha \%))$ values, i.e., that which results in the maximum total number of guaranteed hours per replenishment cycle, as a function of $Q$. In contrast to Figure 10 in which we fix $\hat{h}$ to $\hat{h}^{*}$, to generate Figure 11 we determine $\hat{T}(Q, \hat{h}, \alpha \%)$ for all values of $\hat{h}$ in the set $\{240,300,360,420,480\}$ using equation (6), and then select the pair $(\hat{h}, \hat{T}(Q, \hat{h}, \alpha \%))$ that results in the maximum guaranteed hours per replenishment cycle. (Note that we restrict $\hat{T}(Q, \hat{h}, \alpha \%) \leq 20$.) The resulting pairs are the most favorable ones from the patient perspective (in terms of operation hours per cycle) which simultaneously result in at most $\alpha \%$ loss in coverage. In Figure 11, the value of $\hat{T}(Q, \hat{h}, \alpha \%)$ increases in $Q$ and $\alpha$. Increasing $Q$ or $\alpha$ provides more buffer stock and, consequently, a greater number of sessions becomes acceptable. Interestingly, as seen in Figure 11, 7 hours (i.e., 420 timeslots) is almost consistently the best choice for the number of guaranteed hours. 


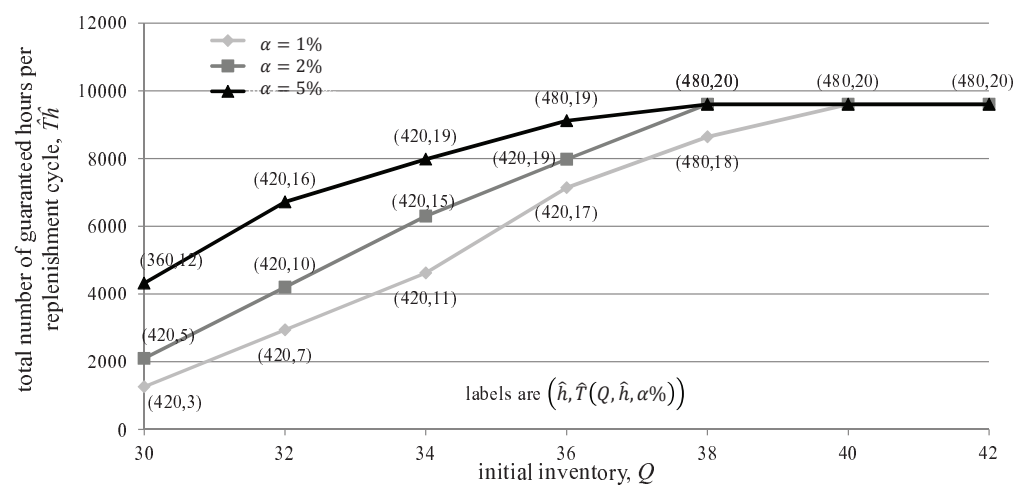

Figure 11: Maximum number of guaranteed hours per replenishment cycle and its corresponding pair of $(\hat{h}, \hat{T}(Q, \hat{h}, \alpha \%))$ as a function of $Q(z=10, \mu T=288$, $1 \leq \hat{T} \leq 20$ and $\hat{h} \in\{240,300,360,420,480\})$.

Another metric by which we can evaluate the performance of a vaccine administration policy is the expected amount of OVW (in doses) per cycle, i.e.,

$$
\text { wastage rate }=\frac{\text { expected amount of OVW (in doses) }}{\text { expected number of doses reconstituted }}
$$

In other words, by wastage rate we mean the expected percentage of doses that are reconstituted and discarded. In Figure 12, we visually assess the relationship between wastage, coverage and the number of sessions. Specifically, Figure 12 shows the wastage rate versus the coverage rate as a function of the number of sessions for three vial sizes. That is, each point corresponds to a different value of $T$, as labeled. For smaller vial sizes, the relationship between wastage and coverage is monotone in $T$, i.e., the fewer the number of sessions, the lower the wastage rate and the higher the coverage rate. When the vial size is 20 , however, the relationship is not monotone, i.e., fewer sessions does not necessarily result in lower wastage or higher coverage. For example, compared to $T=8, T=7$ reduces waste, but also reduces coverage. As marked by the light grey points, some values of $T$ may actually be dominated by others and should therefore not 
be considered for adoption. More specifically a value of 16 sessions performs so well that it dominates $T \in\{10,11,12,13,14,15\}$. That is, compared to $T \in\{10,11,12,13,14,15\}$, $T=16$ offers more frequent sessions while producing less wastage and providing higher coverage.

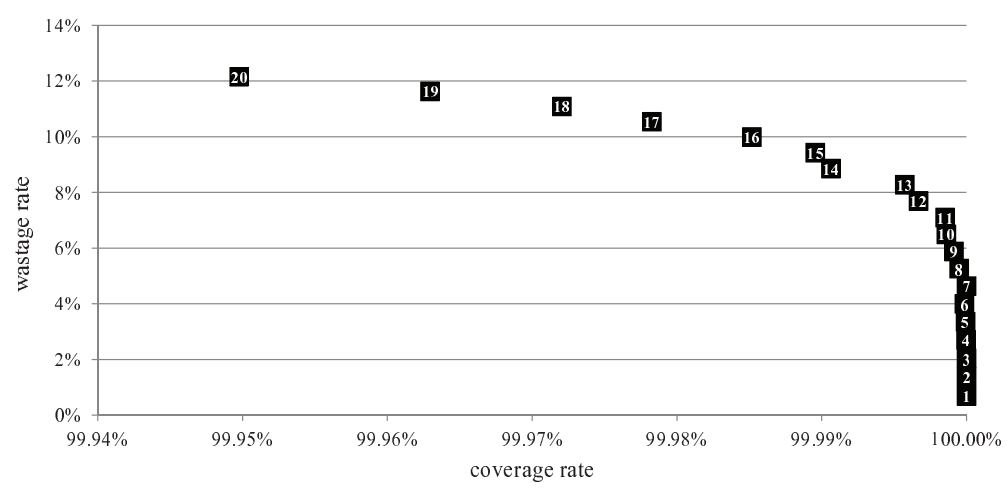

(a) $\mu T=288, Q=72, z=5, \hat{h}=\hat{h}^{*}$.

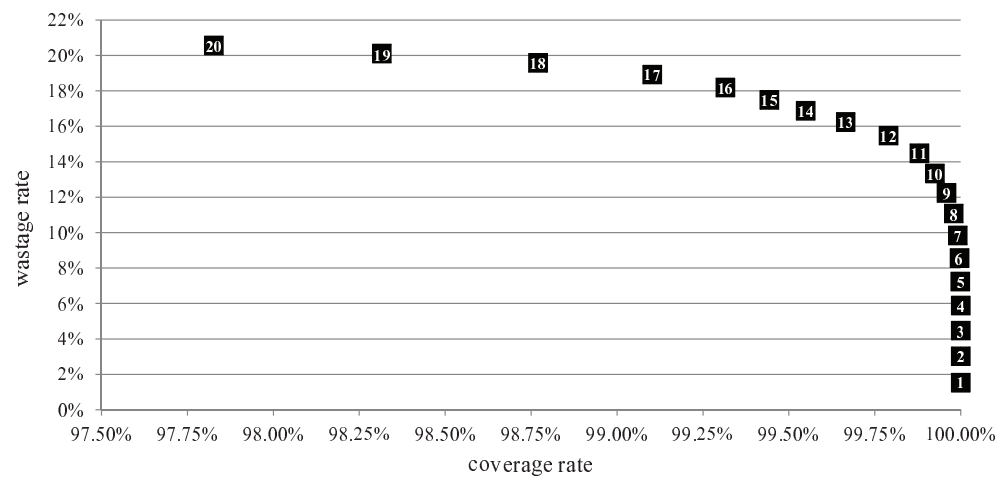

(b) $\mu T=288, Q=36, z=10, \hat{h}=\hat{h}^{*}$.

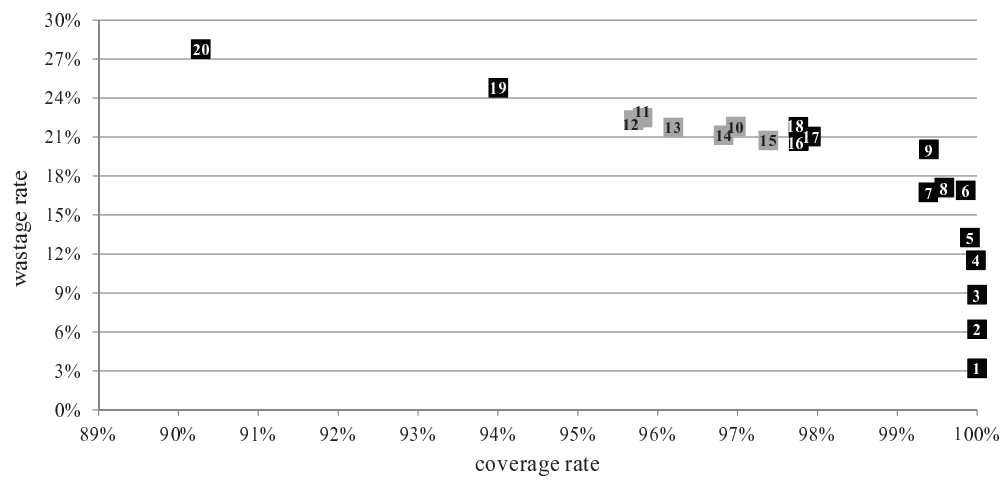

(c) $\mu T=288, Q=18, z=20, \hat{h}=\hat{h}^{*}$.

Figure 12: Open vial wastage rate vs. coverage rate as a function of $T$. 
The unexpected behavior in Figure 12(c) (highlighted in gray) can be explained through the average number of vials available per session, $\frac{Q}{T}$, introduced in Section 3.1. The fractional part of $\frac{Q}{T}$ represents vials which theoretically should be shared between two or more sessions although it is impossible to do in practice. As a result, if the fractional part is farther from 0 or 1 then a larger number of open doses may be discarded. In Figure 12(c) although we decrease the number of sessions from 16 to 11, lower coverage and higher OVW are seen because $\frac{Q}{T}$ moves farther from an integer value as $T$ decreases and given that the vial size is large (20 vs. 5 and 10), the number of unused doses which are discarded at the end of a session is more substantial.

Lastly, we consider the variability of the number of vaccinations under the optimal restricted policy. Variability analysis provides insight into the unpredictability of the number of vaccinations and facilitates worst case analysis. Based on 10,000 replications of the simulation model, we compute a $99 \%$ prediction interval on the number of vaccinations administered. Figure 13 shows the prediction interval for the number of vaccinations under the optimal restricted vaccine administration policy. The variance associated with the number of vaccinations per cycle is rather large due to the high variability in the arrival process, e.g., in Figure 13(b), for $T=1$, the standard deviation of the number of arrivals is 10.7 patients. Furthermore, the variability does not appear to be very sensitive to the number of sessions, especially for larger vial sizes. Depending on the number of sessions, the widths of the $99 \%$ prediction intervals range from (approximately) 50 to 75 vaccinations when using larger vials $(z=20)$, to 35 to 50 vaccinations when using smaller vials $(z=5)$.

\section{Heuristic Policies}

In this section, we consider two heuristic vial administration policies. The first is the dynamic heuristic policy proposed in Mofrad et al. (2014) under which a new vial is opened after guaranteed hours as long as the number of vials remaining is at least as large as the 


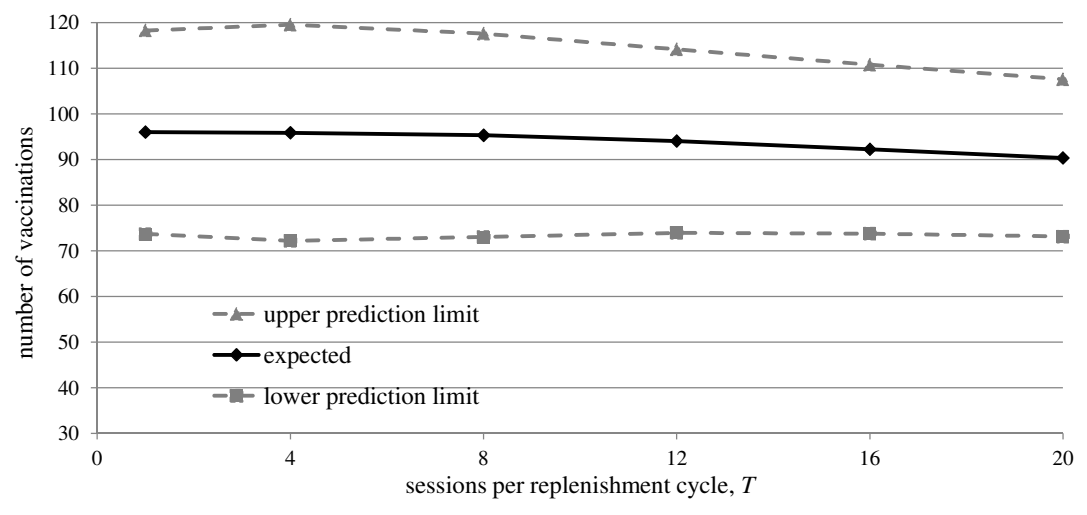

(a) $z=5, Q=24, \mu T=96, \hat{h}=\hat{h}^{*}$.

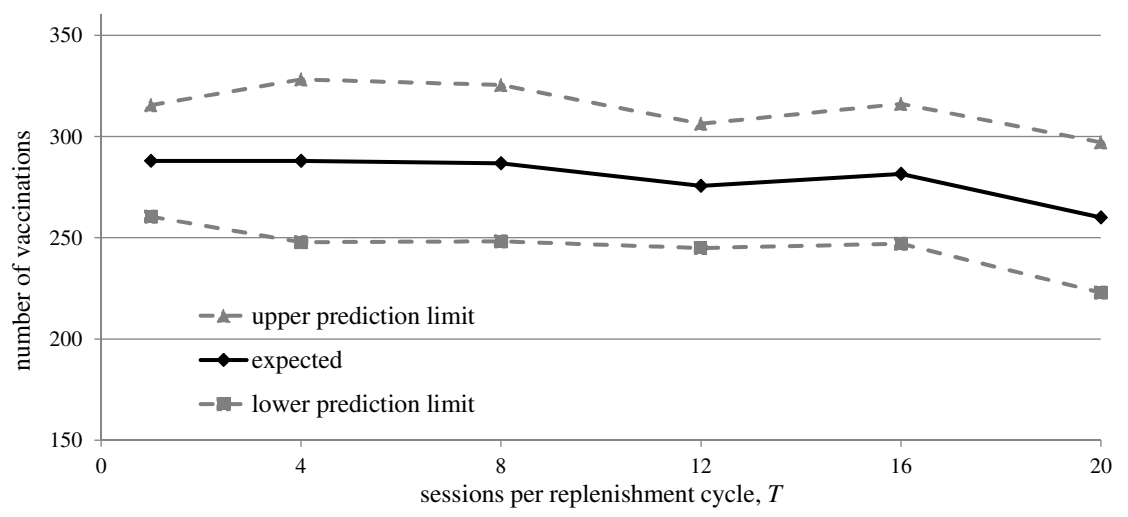

(b) $z=20, Q=18, \mu T=288, \hat{h}=\hat{h}^{*}$.

Figure 13: Prediction interval for the number of vaccinations as a function of the number of sessions per cycle.

number of vials required to satisfy the expected demand in the remaining sessions. The second is a static heuristic policy that specifies a fixed cutoff time after which clinicians do not open new vials. More specifically, this static heuristic approach evaluates all possible cutoff times using the policy evaluation algorithm in Puterman (1994) and selects the one that results in the maximum expected number of vaccinations per replenishment cycle. The resulting static policy is easier to implement than the dynamic heuristic policy proposed in Mofrad et al. (2014) because the same cutoff time is used in each session as opposed to the optimal policy where cutoff points would be different. However, determining the cutoff point of the static heuristic policy requires considerably more computational effort. 
The results suggest that the static heuristic performs very close to the optimal policy and in some cases outperforms the dynamic heuristic. To evaluate the quality of the heuristics, in Table 1 we report the percentage of the gap between the optimal and greedy policies that is covered by the heuristic policies for several problem instances, i.e.,

$$
\text { percentage of gap covered }=\frac{v_{h}-v_{g}}{v^{*}-v_{g}},
$$

where $v^{*}$ is the expected number of vaccinations under the optimal policy (with $\hat{h}=\hat{h}^{*}$ ), $v_{g}$ is the expected number of vaccinations under the greedy policy and $v_{h}$ is the expected number of vaccinations under the heuristic policy. As seen in Table 1, a large portion of the gap is often covered by these policies. For example, for the case in which $T=12$ and $\mu=8$ the percentage of the gap covered by the static and dynamic heuristics is approximately $71 \%$ and $66 \%$, respectively. In Section 5, we study the behavior of these policies more deeply in the presence of random vial yield.

Table 1: The percentage of the gap between the optimal and greedy policies covered by the static and dynamic heuristics $\left(z=10, Q=12, \mu T=96, \hat{h}=\hat{h}^{*}\right)$.

\begin{tabular}{c|c|c|c|c}
\multirow{2}{*}{$T$} & \multirow{2}{*}{$\hat{h}^{*}$} & $\begin{array}{c}\text { cutoff point } \\
\text { (static }\end{array}$ & \multicolumn{2}{|c}{ percentage of gap covered } \\
\cline { 4 - 5 } & & heuristic) & static heuristic & dynamic heuristic \\
\hline 1 & 480 & 480 & $0.0 \%$ & $0.0 \%$ \\
\hline 4 & 390 & 480 & $0.0 \%$ & $72.4 \%$ \\
\hline 8 & 240 & 420 & $71.2 \%$ & $65.9 \%$ \\
\hline 12 & 240 & 300 & $94.5 \%$ & $56.2 \%$ \\
\hline 16 & 90 & 180 & $76.9 \%$ & $62.8 \%$ \\
\hline 20 & 75 & 120 & $84.5 \%$ & $64.5 \%$
\end{tabular}

\section{Impact of Vial-Yield on Policy Performance}

The MDP model proposed in Mofrad et al. (2014) generates an optimal policy under the simplifying assumption of perfect vial yield, i.e., a 5 -dose vial yields exactly 5 doses. 
However, in practice, the number of doses per vial is a random variable due to clinician variation, syringe type and spoilage (i.e., a yield of zero) prior to reconstitution (Griffiths et al. (2011)). In this section, we study the impact of random vial yield on the performance of the optimal policy as well as that of the two proposed heuristic policies defined in Section 4. The results in this section are obtained using the simulation model detailed in Section 2.2.

Let the random variable $Z$ be the number of doses yielded by a multi-dose vial. To analyze the impact of vial yield, we construct a set of stochastically ordered distributions shown in Figure 14. To generate these probability distributions, we consider a fixed set of expected vial yields, $\mathrm{E}[Z] \in\{7,8,9,10,11\}$, and a base probability distribution function from Strauss et al. (2006). To generate the probability distribution associated with each $\mathrm{E}[Z]$, we raise the base cumulative distribution function to the power of the positive number such that it equates to the expected value under the new cumulative distribution and $\mathrm{E}[Z]$.

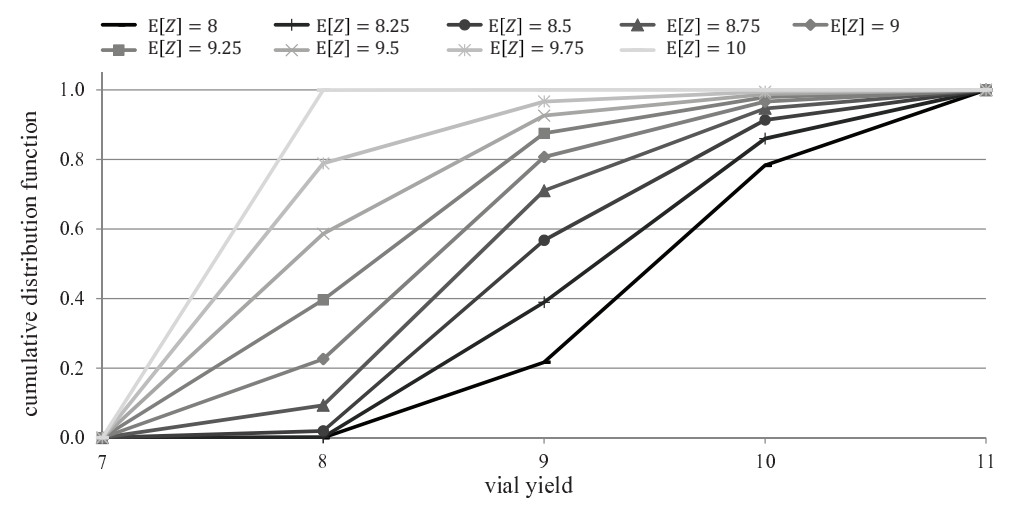

Figure 14: Cumulative distribution functions of vial yield.

In Figure 15, we present the coverage and wastage rates under the optimal policy, the two heuristic policies and the greedy policy for three different vial sizes $(z=10,9$, and 8$)$ as a function of the vial yield distributions shown in Figure 14. We set $z=10,9$ and 8 for the optimal and two heuristic policies because 
the expected vial yield usually falls between 8 and 10. First, as seen in Figure 15(a), the optimal and two heuristic policies derived for a vial size closer to the expected vial size result in a larger coverage rate. For example in Figure 15(b), the coverage rate of the optimal policy with vial size 10 increases from $89.3 \%$ to $97.8 \%$ as $\mathbf{E}[Z]$ increases from 8 to 10 . However, regardless of the expected vial yield, as the vial size decreases the optimal and heuristic policies become more conservative which results in lower wastage rates.

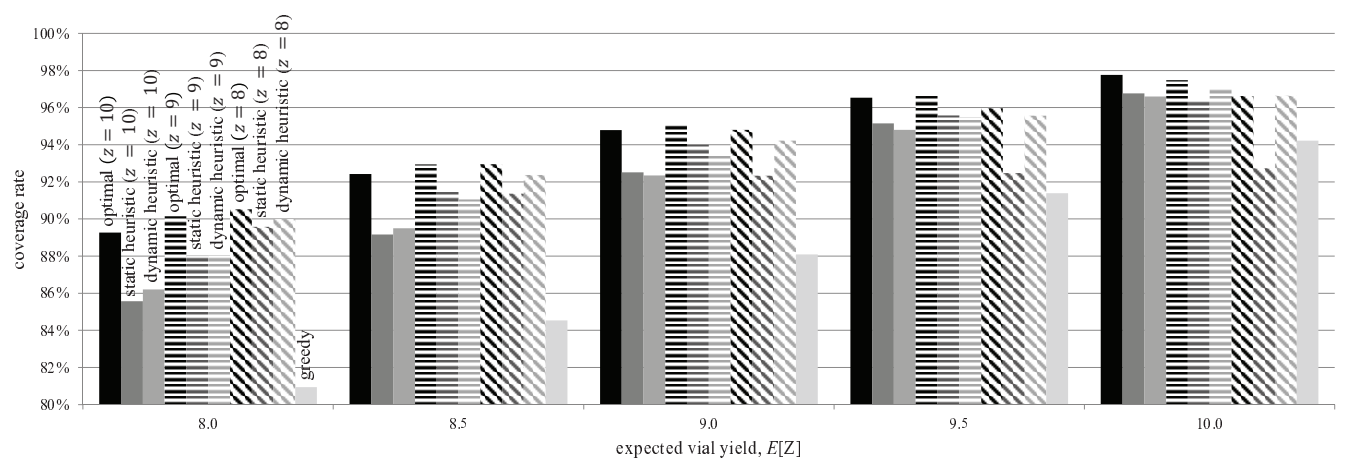

(a) Coverage rate.

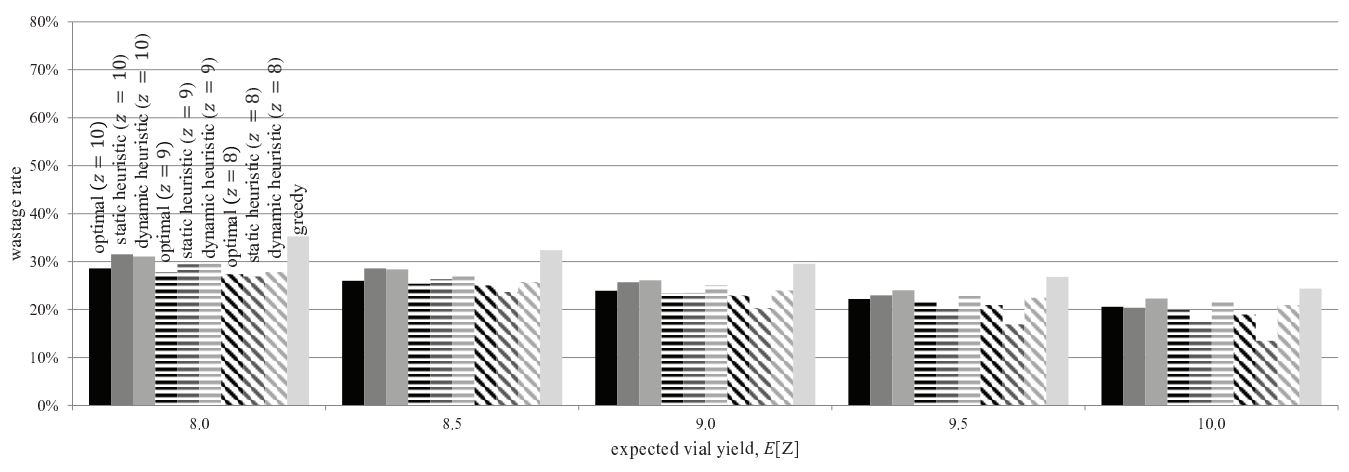

(b) Wastage rate.

Figure 15: Coverage and wastage rate as a function of vial yield distribution in Figure 14 $\left(\mu T=288, T=20, Q=36, z=10, \hat{h}=\hat{h}^{*}\right)$.

Second, the performance of the static and dynamic heuristic policies falls between that of the optimal and greedy policies for $z=10$ and lower than the 
optimal policy for $z=9$ and 8. The greedy policy does not account for vial yield so its performance drops as the expected vial yield decreases. The drop in the performance happens because fewer doses are available and the greedy policy does not adapt to this effective change in the vial size as well as the optimal policy does for a smaller expected vial size than the nominal vial size.

Next, let $p$ be the probability of vial failure due to spoilage. Figure 16 shows the coverage and wastage rate of the optimal policy, the heuristic policies and the greedy policy as a function of the probability of vial failure, $p$. First, as seen in Figure 16, as the probability of vial failure increases, the coverage (wastage) rate of all policies decreases (increases), because a smaller number of vials is available. Second, the wastage rate of the greedy and static heuristic policy are nearly constant as the probability of vial failure increases because these policies have a fixed cutoff time after which clinicians do not open new vials and are therefore independent of the number of vials remaining and subsequently the probability of vial failure. While the optimal and dynamic heuristic policies can better adapt to the loss of vials due to vial failure because in these policies, clinicians make the decision based on the remaining number of vials. Third, the gap between the coverage and wastage rate of the optimal and greedy policies increases in the probability of vial failure. Having a smaller number of vials available results in a more conservative optimal policy, but has no impact on the greedy policy. Therefore, the greedy policy performance deteriorates more rapidly as the probability of failure increases.

\section{Discussion and Cost Implications}

In this section, we begin (Section 6.1) with some general recommendations derived from our analysis of the optimal policy in Sections 3 and 5. In Section 6.2, we estimate the potential reduction in vaccine procurement costs under an optimal policy in three GAVI 


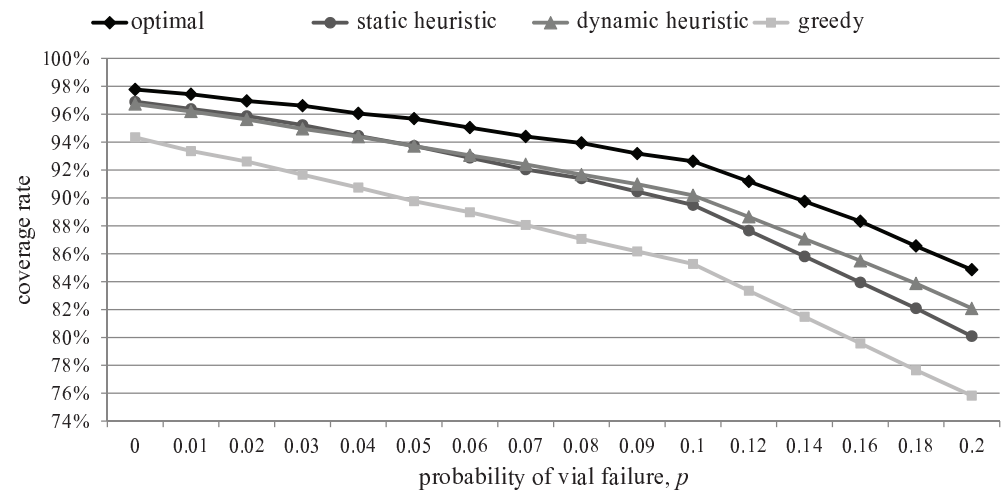

(a) Coverage rate.

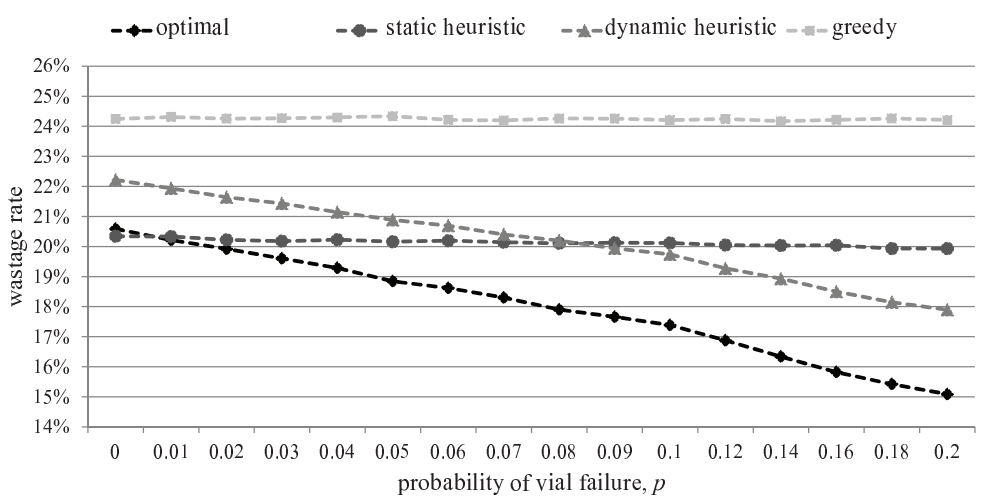

(b) Wastage rate.

Figure 16: Coverage and wastage rate as a function of the probability of vial failure $\left(\mu T=288, T=20, Q=36, z=10, \hat{h}=\hat{h}^{*}\right)$.

countries (Mozambique, Benin and Kenya) and then extrapolate these results to the set of all GAVI countries.

\subsection{Operational Insights}

Clearly, offering more frequent vaccination sessions and guaranteeing longer hours during these sessions is desirable from a patient perspective. However, doing so can also result in more vaccine wastage and lower overall coverage. The analysis in Section 3 shows that these two factors interact with each other, and that the nature of this interaction depends on the vial size and demand rate. Focusing on service to a fixed catchment population, a smaller number of sessions per cycle generally decreases the likelihood of discontinuing service before 
the end of each session and increases the expected number of vaccination hours per session (although not always, as illustrated by Figures 4, 6(a) and 6(b)).

In Section 3.1, we show that a clinic is more likely to have full length sessions when the average number of vials allocated per session $\left(\frac{Q}{T}\right)$ slightly exceeds an integer value. If this fraction is less than one, then the clinic will be forced to skip at least one entire session; therefore, clinic administrators should consider the initial number of vials that they receive during each cycle $(Q)$ to be an upper bound on the number of sessions per cycle. Furthermore, choosing $T$ such that $\frac{Q}{T}$ is slightly larger than a small integer helps maintain longer sessions, even when they are terminated early.

In terms of planned session hours, adopting a large number of guaranteed hours, although desirable from a service perspective, constrains the clinic and can result in poor coverage. The analysis in Section 3.2 shows that there is a relatively clear threshold for guaranteed vaccination hours based on the expected gain in coverage over the greedy policy, especially for larger vial sizes. However, as seen in Figures $7(\mathrm{a})$ and $7(\mathrm{~b})$, the threshold value can vary widely, ranging from 300 - 400 minutes for typical parameter values during a 480-minute session. Forcing the clinic to guarantee hours beyond this threshold can lead to degradation in coverage, however, guaranteeing fewer hours does not significantly improve coverage.

As discussed in Section 3.3, although having a larger number of sessions can increase patient convenience, it can also decrease the coverage rate. The "best" scenario in terms of maximizing coverage would be a single session per cycle that all patients in the catchment area attend. Of course, this scenario is not practical; however, it does provide an upper bound on the expected coverage. Increasing the number of sessions per cycle can therefore be evaluated with respect to this bound in terms of the percentage loss in the expected number of vaccinations, and clinicians can increase the number of sessions as long as this loss is not unacceptably large. Alternatively, guaranteed hours and session frequency can be considered simultaneously with a goal of maximizing hours of operation subject 
to a similar limit on percentage loss. The analysis in Section 3.3 suggests that clinics should prioritize increasing the number of guaranteed hours per session as opposed to the number of sessions per cycle to maximize this measure of patient convenience.

It should be mentioned that trying to predict the exact number of vaccinations that a clinic can provide for a given vial size and a given number of sessions per replenishment cycle is difficult. There can be considerable variability, especially when using larger vial sizes. Thus, clinicians should not be alarmed if they see some variation with respect to the number of vaccinations provided across different replenishment cycles. Finally, in Section 5 , we show that in the presence of random vial yield, implementing the optimal policy for a vial size close to the expected vial yield results in better coverage than merely using the nominal vial size value (Figure 15).

Though we have been able to provide some general guidelines for vaccine administration, our computational results also indicate that the operating strategies that maximize vaccine coverage often depend heavily on the characteristics of the clinic. Therefore, we have developed a decision support tool to permit users to conduct analysis similar to that performed in Sections 3 and 5 for their particular setting. The tool is called the Vaccine Clinic Reconstitution Optimizer (VaCRO). VaCRO develops a vaccine adminstration policy based on the MDP model in Mofrad et al. (2014). We have made VaCRO freely available online for download (Vaccine Clinic Reconstitution Optimizer (2015)) and promoted it via The Technical Network for Strengthening Immunization Services (TechNet-21 (2015)), an on-line forum for global health logisticians and others interested in improving immunization programs and their effectiveness. 


\subsection{Cost Analysis}

In this section, we estimate the reduction in procurement costs associated with switching from the greedy policy to an optimal vial allocation policy. To do so, we use available data for the annual birth rate of clinics in three GAVI countries - Mozambique, Benin and Kenya. Because of the large variation in the clinic birth rates across these countries, we divide the clinics into three sizes - small, medium, and large. Clinics with less than 365 births per year (i.e., one birth per day) are classified as small clinics, clinics with at least 365 births per year and at most 1095 births per year (i.e., 3 births per day) are classified as medium clinics and the remaining clinics are classified as large clinics. We assume a constant birth rate over the year; monthly replenishment in all clinics; and as an illustrative vaccine, we choose measles, which requires 2 doses per child. We then estimate the monthly demand using the average monthly birth rates of the clinics in the middle $80 \%$ of each size group. In Table 2, we report the estimated monthly demand for each clinic size.

Table 2: Average number of arrivals per replenishment cycle in Mozambique, Benin and Kenya for a two-dose measles vaccine schedule.

\begin{tabular}{c|c|c|c|c}
\multirow{2}{*}{ country } & \multirow{2}{*}{$\begin{array}{c}\text { total number of } \\
\text { clinics }\end{array}$} & \multicolumn{3}{|c}{ average number of arrivals per month } \\
\cline { 3 - 5 } & & small clinics & medium clinics & large clinics \\
\hline Mozambique & 114 & 31.4 & 95.5 & 284.8 \\
\hline Benin & 658 & 43.1 & 97.9 & 260.7 \\
\hline Kenya & 2733 & 34.3 & 94.4 & 348.9
\end{tabular}

To compare the performance of the greedy and optimal policy, we set the minimum coverage rate to be $95 \%$ and compute the required initial inventory under each policy using the monthly demand data presented in Table 2 and parameter values stated in Table 3. We then compute the reduction in the procurement cost per vaccination realized by switching to the optimal policy. Finally, using a weighted average based on the proportion of patients who fall into each clinic size in each country, we compute the average reduction in the procure- 
ment cost per vaccination for each country. The resulting reduction in procurement costs per vaccination in Mozambique, Benin and Kenya are approximately $\$ 0.051 /$ administered dose, $\$ 0.033 /$ administered dose and $\$ 0.031 /$ administered dose, respectively, which are equivalent to $13.8 \%, 9.1 \%$ and $8.7 \%$ of the procurement cost per vaccination under the greedy policy.

Table 3: Parameter values used in the cost analysis of the optimal and greedy $\operatorname{policy}\left(\hat{h}=\hat{h}^{*}\right)$.

\begin{tabular}{|c|c|c|c|c|c|c|c|c|c|c|c|c|c|c|c|c|c|}
\hline \multirow{2}{*}{$\begin{array}{l}\text { vaccination } \\
\text { policy }\end{array}$} & \multirow{2}{*}{ country } & \multirow{2}{*}{$\begin{array}{r}\text { buffer } \\
\text { stock }\end{array}$} & \multicolumn{5}{|c|}{ small-size clinics } & \multicolumn{5}{|c|}{ medium-size clinics } & \multicolumn{5}{|c|}{ large-size clinics } \\
\hline & & & $\mu$ & $Q$ & $z$ & $T$ & $\hat{h}$ & $\mu$ & $Q$ & $z$ & $T$ & $\hat{h}$ & $\mu$ & $Q$ & $z$ & $T$ & $\hat{h}$ \\
\hline \multirow{3}{*}{$\begin{array}{c}\text { optimal } \\
\text { policy }\end{array}$} & Mozambique & \multirow{3}{*}{$25 \%$} & 7.85 & 4 & \multirow{3}{*}{10} & \multirow{3}{*}{4} & \multirow{3}{*}{240} & 7.96 & 12 & \multirow{3}{*}{10} & 12 & 240 & 14.24 & 33 & \multirow{3}{*}{10} & \multirow{3}{*}{20} & 345 \\
\hline & Benin & & 10.78 & 6 & & & & 8.16 & 13 & & 12 & 255 & 13.03 & 31 & & & 330 \\
\hline & Kenya & & 8.58 & 5 & & & & 7.87 & 12 & & 12 & 255 & 17.44 & 39 & & & 375 \\
\hline \multirow{3}{*}{$\begin{array}{l}\text { greedy } \\
\text { policy }\end{array}$} & Mozambique & \multirow{3}{*}{$25 \%$} & 7.85 & 5 & \multirow{3}{*}{10} & \multirow{3}{*}{4} & \multirow{3}{*}{480} & 7.96 & 14 & \multirow{3}{*}{10} & \multirow{3}{*}{12} & \multirow{3}{*}{480} & 14.24 & 37 & \multirow{3}{*}{10} & \multirow{3}{*}{20} & \multirow{3}{*}{480} \\
\hline & Benin & & 10.78 & 7 & & & & 8.16 & 14 & & & & 13.03 & 34 & & & \\
\hline & Kenya & & 8.58 & 5 & & & & 7.87 & 14 & & & & 17.44 & 42 & & & \\
\hline
\end{tabular}

Next, we extrapolate these results to all GAVI countries. To do so, we first estimate the average reduction in the procurement cost per vaccination when the optimal policy is adopted in all GAVI countries using a weighted average of the reductions in Mozambique, Benin and Kenya. More specifically, we use the newborn rate of each country as its corresponding weight and estimate $\left(\frac{51826 \times \$ 0.051+371022 \times \$ 0.033+1516221 \times \$ 0.031}{51826+371022+1516221} \approx\right) \$ 0.032 /$ administered dose reduction in procurement costs on average in all GAVI countries. We then estimate a $(72,952,312 \times \$ 0.032 \times 2 \approx) \$ 4.6$ million reduction in procurement costs per year across all the GAVI countries given that 2 doses are required per child and the total birth cohort of all GAVI countries is approximately 73 million (Global Alliance for Vaccines and Immunization (2014)). It is important to note that this cost reduction is computed for measles which is a relatively inexpensive vaccine; other types of vaccines are often 10 times (or more) as expensive. Therefore, this $\$ 4.6$ million savings is a conservative estimate of the reduction in procurement costs per vaccine type. 


\section{Limitations and Future Work}

In this section, we address some of the limitations of our analysis and present directions for future work. From a modeling perspective, the MDP model in Mofrad et al. (2014) assumes that arrival rates during and across sessions are stationary and that patients who are not vaccinated are lost. Modeling these types of nonstationarities, patient returns and other adaptive behaviors of patients, adds considerable complexity to the MDP model and is left for future work.

Due to data scarcity, we evaluate proposed recommendations and criteria using representative problem instances. Although the parameter values selected are representative of values observed in practice, performing empirical studies regarding patient arrival patterns would allow us to gain better understanding on how to inform the models and generate recommendations.

Additionally, we focus on in-clinic vaccine administration in isolation. In reality, in-clinic operations and outreach strategies are often linked together by a shared supply of vaccines and healthcare workers. Consequently, further analysis on their interaction and its impact on vaccine and healthcare worker allocation is desirable.

\section{Acknowledgment}

This work has been supported by National Science Foundation Grant No. CMMI-1131172.

\section{References}

Assi, T.-M., Brown, S.T., Djibo, A., Norman, B.A., Rajgopal, J., Welling, J.S., Chen, S.-I., Bailey, R.R., Kone, S., Kenea, H., et al. 2011. Impact of changing the measles vaccine vial 
size on Niger's vaccine supply chain: A computational model. BMC Public Health, 11(1), 425.

Center for Disease Control and Prevention. 2007. Vaccine management: Recommendations for handling and storage of selected biologicals. US Department of Health and Human Services, Public Health Service, Centers for Disease Control.

Dhamodharan, A., \& Proano, R.A. 2012. Determining the optimal vaccine vial size in developing countries: A Monte Carlo simulation approach. Health Care Management Science, 15(3), 188-196.

Drain, P.K., Ralaivao, J.S., Rakotonandrasana, A., \& Carnell., M.A. 2003. Introducing auto-disable syringes to the national immunization programme in Madagascar. Bulletin of the World Health Organization, 81(8), 553-560.

Global Alliance for Vaccines and Immunization. 2014. Country hub. Available from http: //www.gavialliance.org/country/, information and data accessed on July 14, 2014.

Griffiths, U.K, Santos, A.C., Nundy, N., Jacoby, E., \& Matthias, D. 2011. Incremental costs of introducing jet injection technology for delivery of routine childhood vaccinations: Comparative analysis from Brazil, India, and South Africa. Vaccine, 29(5), 969-975.

Lee, B.Y., Norman, B.A., Assi, T.M., Chen, S.I., Bailey, R.R., Rajgopal, J., Brown, S.T., Wiringa, A.E., \& Burke, D.S. 2010. Single versus multi-dose vaccine vials: An economic computational model. Vaccine, 28(32), 5292-5300.

Lee, B.Y., Assi, T.M., Rookkapan, K., Connor, D.L., Rajgopal, J., Sornsrivichai, V., Brown, S.T., Welling, J.S., Norman, B.A., Chen, S.I., Bailey, R.R., Wiringa, A.E., Wateska, A.R., Jana, A., Van Panhuis, W.G., \& Burke, D.S. 2011. Replacing the measles ten-dose vaccine presentation with the single-dose presentation in Thailand. Vaccine, 29(21), 3811-3817. 
Merck \& Co., Inc. 2014. M-M-R II (measles, mumps, and rubella vaccine live). Available from https://www.merck.com/product/usa/pi_circulars/m/mmr_ii/mmr_ii_pi.pdf, information and data accessed on September 28, 2015.

Mofrad, M.H., Maillart, L.M., Norman, B.A., \& Rajgopal, J. 2014. Dynamically optimizing the administration of vaccines from multi-dose vials. IIE Transactions, 46(7), 623-635.

Parmar, D., Baruwa, E.M., Zuber, P., \& Kone, S. 2010. Impact of wastage on single and multi-dose vaccine vials: Implications for introducing pneumococcal vaccines in developing countries. Human Vaccines, 6(3), 270-278.

Puterman, M.L. 1994. Markov Decision Processes: Discrete Stochastic Dynamic Programming. John Wiley and Sons.

Strauss, K., van Zundert, A., Frid, A., \& Costigliola, V. 2006. Pandemic influenza preparedness: The critical role of the syringe. Vaccine, 24(22), 4874-4882.

TechNet-21. 2015. VaCRO: Vaccine Clinic Reconstitution Optimizer. Available from http:// technet-21.org/en/forums/vacro-vaccine-clinic-reconstitution-optimizer accessed on September 30, 2015.

Vaccine Clinic Reconstitution Optimizer. 2015. VaCRO. Available from http://www.pitt. edu/ vacro/ accessed on September 30, 2015.

World Health Organization. 2005. Monitoring vaccine wastage at country level: Guidelines for programme managers.

Yang, W., Parisi, M., Lahue, B.J., Uddin, M.J., \& Bishai, D. 2014. The budget impact of controlling wastage with smaller vials: A data driven model of session sizes in Bangladesh, India (Uttar Pradesh), Mozambique, and Uganda. Vaccine, 32(49), 6643-6648. 